\title{
A Morphological Study of Dorsalis Pedis Artery and Its Clinical Correlation
}

\author{
Dr.Vasudha Kulkarni ${ }^{1}$, Dr.B.R.Ramesh ${ }^{2}$ \\ ${ }^{1,2}$ (Department of Anatomy, Dr. B. R. Ambedkar medical college/ Rajiv Gandhi University of Health Sciences, \\ Karnataka, India)
}

\begin{abstract}
Dorsalis Pedis artery is the artery for peripheral vascular disease. It is the direct continuation of anterior tibial artery. Evaluation of Dorsalis pedis artery pulsations is an useful clinical tool for assessing peripheral arterial perfusion. The present study aims at determining branching pattern of Dorsalis pedis artery. The objective is to provide data on thediameter of Dorsalis pedis artery and its branching pattern. A study of Dorsalis pedis artery was done in thirty three lower limbs at Dr.B.R.Ambedkar medical college by dissection method. The diameter of Dorsalis pedis artery at its origin varied from 1.5 to $5 \mathrm{~mm}$. Maximum diameter is 5mm, minimum is $1.5 \mathrm{~mm}$. Average is $3.31 \mathrm{~mm}$. Arterial branching pattern is categorised into A, $B$, $C, D$ and $E$. The study infers correlation between diameter of Dorsalis pedis Artery and its branching pattern. This study also provides data for vascular mapping of foot prior to certain surgeries like free flap transplantation.
\end{abstract}

Keywords: Artery of dorsum of foot, Arteria dorsalis pedis, Ankle brachial index, Dorsalis pedis artery, Dorsal artery of foot, Pedal artery, Peripheral pulse

\section{Introduction}

Arteria Dorsalis pedis / Dorsalis pedis artery is the prime source of vascular irrigation of dorsum of foot. It is the artery of peripheral vascular disease. Dorsalis pedis artery is an easily accessibleartery for assessing pedal pulsations [1].Examination of pedal pulses remains a useful clinical tool when evaluating peripheral circulationand absence of the Dorsalis pedis pulsation in a child can lead to contracture and retard trophic growth of foot [2]. The anatomical basis for the absence of its pulsations is the change in the arterial branching pattern, deviant course and its small caliber [3].The present study is done to determine the branching pattern and caliber of the artery. The study is germane to vascular surgeons for understanding the degree of stenosis of the artery in peripheral vascular disease while performing endovascular catheterization.Thus awareness of the anatomical variability as determined by vascular development is imperative for radiologists and plastic surgeons [4].

\subsection{Relevant Anatomy}

Dorsalis pedis artery is the continuation of anterior tibial artery distal to ankle joint, extending along an imaginary line drawn from midpoint of transverse bimalleolar axis to tip of first metatarsal space. It courses along the tibial side of the foot up to proximal end of $1^{\text {st }}$ inter - metatarsal space, passes between two heads of first dorsal interosseous muscle and completes the plantar arch with lateral plantar artery. Its branches are medial and lateral malleolar arteries, artery to sinus tarsi, lateral tarsal artery, first dorsal metatarsal artery and arcuate artery [5].

\subsection{Aims and Objectives}

Aim of the present study is to determine arterial pattern of Dorsalis pedis artery. Objective is to provide data of diameter at origin of Dorsalis pedis arteryand to find its association with branching pattern.

\section{Methodology}

After the approval by Institutional Ethical committee, dissecting room cadavers allotted for MBBS students were selected for the study. Thirty three lower limb specimens were selected and dorsum of foot was dissected.

\subsection{Inclusion criteria}

The specimens included all the adult lower limbs with intact Dorsalis pedis artery.

\subsection{Exclusion criteria}

Newborn specimens were excluded. Conical enlargements if present at the point of origin and along the course of artery were excluded. 


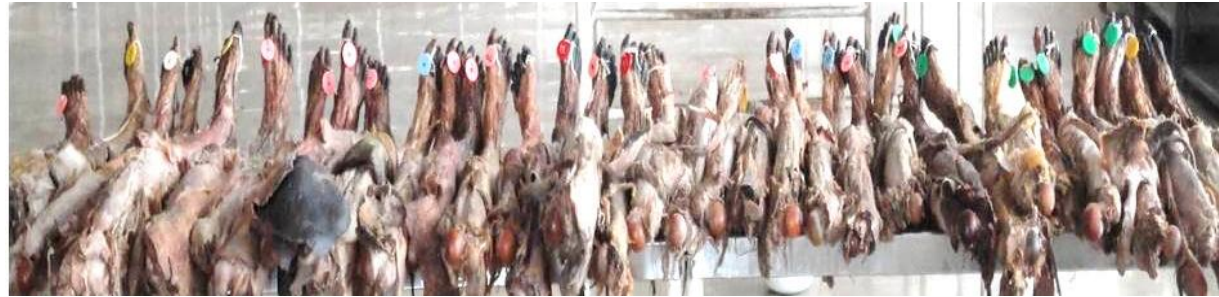

Figure 1 showing display of thirty three adult lower limb specimens

Dorsalis pedis artery was traced from its origin in the mid-malleolar region to its termination at the proximal end of the first metatarsal space. Its branching pattern was determined and photographed.The data was recordedusing Vernier calipers for a) level of origin, b) diameter of Dorsalis pedis artery at origin, c)diameter of first dorsal metatarsal artery at its origin and d)variation in branching pattern of Dorsalis pedis artery.

\section{Results}

Out of thirty three lower limb specimens, twenty are right limbs $(60.6 \%)$ and thirteen are left limb specimens $(39.4 \%)$. The diameter of dorsalis pedis artery at its origin is in the range of $1.5 \mathrm{~mm}$ to $5 \mathrm{~mm}$. The mean diameter at origin is $3 \mathrm{~mm}$ in $33.3 \%$ of cases(11 out of 33 limbs) followed by $4 \mathrm{~mm}$ in $24.2 \%$ (8 out of 33 limbs), $5 \mathrm{~mm}$ in $15.2 \%$ of cases(5 out of $33 \mathrm{limbs}$ ) and $2 \mathrm{~mm}$ in $6.1 \%$ (2 out of 33limbs). Least common diameter of Dorsalis pedis artery at its origin is $1.5 \mathrm{~mm}$ and $3.5 \mathrm{~mm}$ in $3 \%$ cases each ( 3 out of $33 \mathrm{limbs}$ ). The diameter of first dorsal metatarsal artery at its origin ranged from $1 \mathrm{~mm}$ to $3 \mathrm{~mm}$. Mean diameter is $2 \mathrm{~mm}$ in $27.3 \%$ (9 out of 33 limbs). The first dorsal metatarsal artery is absent in $33.3 \%$ of cases(11 out of 33 limbs).Branching pattern of dorsalis pedis artery was dissected, observed and classified into five types [3]

Type A $(15.2 \%)$ - Usual pattern of arterial tree of dorsum of foot. Dorsalis pedis artery along its course divided into lateral and medial tarsal artery, first dorsal metatarsal artery and arcuate artery (Fig 2A).

Type B $(9.1 \%)$-Anterior tibial artery divides into a larger lateral tarsal artery and a smaller dorsalis pedis artery. The latter is of small caliber and lateral tarsal artery is larger, easily palpable. Dorsalis pedis artery continues downwards over talus, navicular, cuneiform bones and then between $1^{\text {st }}$ and $2^{\text {nd }}$ metatarsal bones, it gives off of its branch first dorsal metatarsal artery and branches to first and second toes. Over lateral cuneiform bone, arcuate artery is a branch of lateral tarsal artery (Fig 2B).

Type C(42.4\%)- Dorsalis pedis artery is absent as an independent vessel. It becomes merely a loop in the arterial pattern of the dorsum (Fig 2C).

Type D (6.1\%)- Reduction of anterior tibial artery and its branches and increasing participation of arteries of sole in the supply of dorsum. Dorsalis pedis artery is not recognizable even as a loop and anterior tibial artery continues downwards as a central channel with reduced size. The plantar arteries supply dorsum predominantly. Pulses are not palpable clinically in such cases (Fig 2D).

Type E $(12.1 \%)$ - Increasing participation of anterior peroneal branch from posterior tibial artery. Plantar tibial vessels still take a prominent part in arterial supply. Dorsalis pedis artery along with its branch lateral tarsal artery is not clinically palpable (Fig 2E).

In addition to these types, in 2 specimens, dorsalis pedis artery exhibited lateral deviation (Fig 2DC).

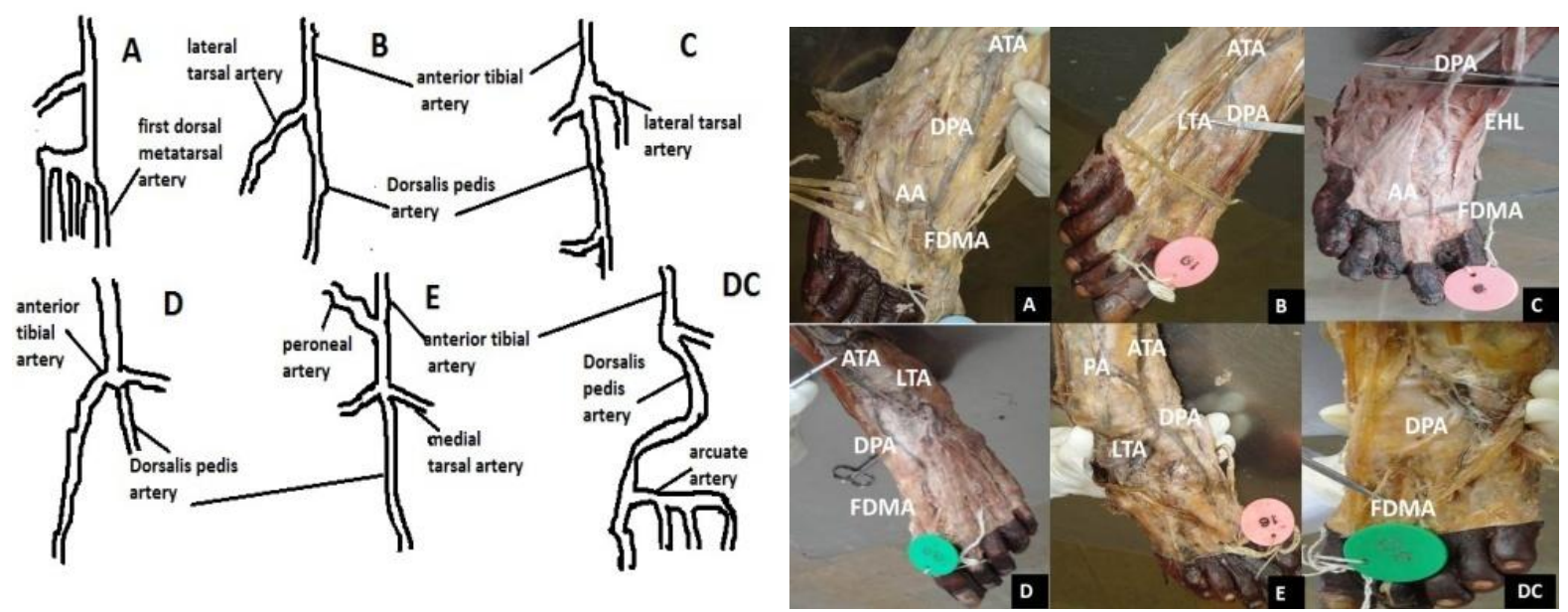

Fig 2 A- E Types of variations of dorsalis pedis artery, Fig DC - Lateral deviation of dorsalis pedis artery, schematicrepresentation is indicated on the left side. Anterior tibial artery(ATA), Dorsalis Pedis artery(DPA), Lateral tarsal artery (LTA), First dorsal metatarsal artery(FDMA), Peroneal artery (PA), Arcuate $\operatorname{artery}(\mathrm{AA})$, Extensor hallucis longus(EHL) 


\subsection{Data analysis}

Statistical analysis was done to find correlation between diameter of dorsalis pedis artery and its branching pattern. The results are tabulated.

Table 1 showing percentage of diameters of dorsalis pedis artery at origin

\begin{tabular}{|c|c|c|c|c|c|c|c|c|c|}
\hline \multicolumn{2}{|c|}{$\begin{array}{c}\text { Diameter at } \\
\text { origin(in } \\
\text { mm) }\end{array}$} & \multirow{2}{*}{$\begin{array}{c}\text { Frequency } \\
5\end{array}$} & \multirow{2}{*}{$\begin{array}{c}\text { Percent } \\
15.2\end{array}$} & \multirow{2}{*}{$\begin{array}{c}\begin{array}{c}\text { Valid } \\
\text { Percent }\end{array} \\
15.2\end{array}$} & \multirow{2}{*}{$\begin{array}{c}\begin{array}{c}\text { Cumulative } \\
\text { Percent }\end{array} \\
15.2\end{array}$} & \multicolumn{4}{|c|}{ Chi-Square Tests } \\
\hline \multirow{8}{*}{ Valid } & $0-1.4$ & & & & & & Value & $\begin{array}{l}\text { Degrees } \\
\text { of } \\
\text { freedom }\end{array}$ & $\begin{array}{l}\text { Asymp. } \\
\text { Sig. } \\
\text { (2-sided) }\end{array}$ \\
\hline & $\begin{array}{l}1.5- \\
1.9\end{array}$ & 1 & 3.0 & 3.0 & 18.2 & $\begin{array}{l}\text { Pearson } \\
\text { Chi- } \\
\text { Square }\end{array}$ & $65.755^{\mathrm{a}}$ & 30 & .000 \\
\hline & $2-2.9$ & 2 & 6.1 & 6.1 & 24.2 & $\begin{array}{l}\text { Likelihood } \\
\text { Ratio }\end{array}$ & 49.753 & 30 & .013 \\
\hline & $3-3.4$ & 11 & 33.3 & 33.3 & 57.6 & $\begin{array}{l}\text { No of } \\
\text { Valid } \\
\text { Cases } \\
\end{array}$ & 33 & & \\
\hline & $\begin{array}{l}3.5- \\
3.9 \\
\end{array}$ & 1 & 3.0 & 3.0 & 60.6 & & & & \\
\hline & $\begin{array}{l}4- \\
4.9 \\
\end{array}$ & 8 & 24.2 & 24.2 & 84.8 & & & & \\
\hline & 5 & 5 & 15.2 & 15.2 & 100.0 & & & & \\
\hline & Total & 33 & 100.0 & 100.0 & & & & & \\
\hline
\end{tabular}

Table 2 showing relation between branching pattern and diameter at origin of dorsalis pedis artery

\begin{tabular}{|l|l|l|l|l|l|l|l|l|l|}
\hline Branching pattern * Diameter at origin Cross tabulation \\
\hline & & \multicolumn{2}{l|}{ Diameter at origin } \\
\hline & & 0 & 1.5 & 2 & 3 & 3.5 & 4 & 5 & \\
\hline \multirow{3}{*}{$\begin{array}{l}\text { Branching } \\
\text { pattern }\end{array}$} & 0 & 5 & 0 & 0 & 0 & 0 & 0 & 0 & 5 \\
\cline { 2 - 11 } & $\mathrm{A}$ & 0 & 0 & 1 & 3 & 0 & 0 & 1 & 5 \\
\cline { 2 - 11 } & $\mathrm{B}$ & 0 & 0 & 0 & 2 & 0 & 1 & 0 & 3 \\
\cline { 2 - 10 } & $\mathrm{C}$ & 0 & 0 & 1 & 4 & 0 & 5 & 4 & 14 \\
\cline { 2 - 10 } & $\mathrm{D}$ & 0 & 1 & 0 & 0 & 0 & 1 & 0 & 2 \\
\cline { 2 - 9 } & $\mathrm{E}$ & 0 & 0 & 0 & 2 & 1 & 1 & 0 & 4 \\
\hline Total & 5 & 1 & 2 & 11 & 1 & 8 & 5 & 33 \\
\hline
\end{tabular}

\section{Discussion}

Variations of the arteries of lower limb have been widely studied by anatomists, radiologists and surgeons. These arteries not only create anatomical interest but they also have profound clinical importance. In the present study, thirty three lower limb specimens were studied for determining diameter and arterial pattern of dorsalis pedis artery.

Presence of pedal pulsation rules out circulatory disorders on the contrary, its absence aids in the diagnosis of peripheral vascular disease[6].Extra circulatory factors such as oedema, adiposity, ligaments may also diminish pedal pulsations and pose diagnostic errors[3]. Doppler ultrasonography is non-invasive tool for evaluation of peripheral vascular disease. Peak to peak pulsatility index (peak to peak frequency difference of Doppler waveform divided by mean frequency) is greatest in dorsalis pedis and posterior tibial arteries. Digital subtraction contrast arteriography remains the gold standard for identifying definitive arterial anatomy of dorsalis pedis artery during trans metatarsal amputation with plantar flap and dorsalis pedis artery bypass[6].

Absence of pulsations of Dorsalis pedis artery in normal individuals as reported by various authors: 
A Morphological study of Dorsalis pedisartery and its Clinical Correlation

Table showing incidence of absence of dorsalis pedis pulse as reported by various authors[1,2,3,6,7,8 ].

\begin{tabular}{|c|c|c|}
\hline Sl no & Authors & Percentage ofabsence of dorsalispedis pulse \\
\hline 1 & Cronenwett & $10 \%$ \\
\hline 2 & Reich & $4.2 \%$ \\
\hline 3 & Kennedy Legel & $10 \%$ \\
\hline 4 & Demetrios Chavatzas & $2.25 \%$ \\
\hline 5 & Vijayalakshmi & $10-12 \%$ \\
\hline 6 & Steven G & $12 \%$ \\
\hline 7 & Erb & $2 \%$ \\
\hline 8 & Rudolph Reich & $4 \%, 8 \%$ (Abnormal position) \\
\hline
\end{tabular}

\subsection{Embryological significance}

The lower limb buds are supplied by branches of the inter-segmental arteries which arise from aorta and form fine capillary network throughout the mesenchyme[1]. The primordial vascular pattern consists of a primary axial artery and its branches. The arteries of lower limb arise from two sources; the primary limb artery /axis artery (Sciatic artery) and femoral artery.Sciatic artery arises from the dorsal root of the umbilical artery and runs through the thigh, knee and leg. By $14 \mathrm{~mm}$ embryonic stage, the femoral artery grows into the thigh, joins the sciatic artery.Below knee, the latter lies between tibia and popliteus. The proximal part then regresses. Primitive middle and distal segments of the sciatic artery persists to form definitive popliteal and peroneal arteries[9].

At the distal border of the popliteus, a perforating branch that communicates with the femoral artery arises from the sciatic artery. This branch called as Ramus communicans forms anterior tibial artery which passes ventrally between tibia and fibula. Dorsalis pedis artery is the distal continuation of anterior tibial artery.Popliteal and early distal femoral artery anastomoses to form posterior tibial artery[9].

Variations in branching pattern of dorsalis pedis artery could be explained by functionaldominance of its branches. Tiny blood vessels are derived from the blood islands at $3^{\text {rd }}$ or $4^{\text {th }}$ week of development. They merge with each other forming a continuous network from which buds grow out, canals are formed and new vessels are thus formed. Depending on the functional dominance some vessels regress, diverge in the mode of origin course from the principal vessel[1].

\subsection{Phylogenetic history}

Anterior and posterior tibial arteries are special features derived from the anastomosis of muscular vessels which carry the popliteal circulation into the lower leg. In primates, the femoral artery is continued characteristically into the leg as the saphenous artery which accompanies the saphenous nerve and great saphenous vein to the region of the ankle. The saphenous artery descends quite superficially on the medial surface of knee and leg. The saphenous artery gives off a posterior branch which descends behind medial malleolus, giving origin to medial and lateral plantar arteries. The medial plantar artery is theearliest amongst the two. The saphenous artery itself continues as the dorsalis pedis artery[2].

After differentiation of the posterior tibial artery as a connecting link between popliteal and plantar vessels the peroneal artery makes its appearance by the continuity of an anastomotic chain. In the same manner, at a later stage, anterior tibial artery develops from an anastomotic chain connecting popliteal artery with dorsalis pedis artery. This new vessel reaches its full development in human being alone and saphenous artery is lost[2].

Thus, due to relatively recent transformation in vascular pattern of the human leg and foot, the arterial channels of the dorsum present variable character.

\subsection{Clinical significance}

Major vessel to talus is dorsalis pedis artery and its branches. These are distributed mainly at the junction of neck and the body of the talus in the tarsal sinus area. The branches to talus arise at the point where anterior tibial artery continues as dorsalis pedis artery. The artery to tarsal sinus arises from an anastomotic loop between lateral tarsal branch of dorsalis pedis artery and perforating branch of peroneal artery. Lateral tarsal artery may also give direct branches to the head of the talus and to the anterolateral part of the body of talus. The knowledge of vascular course of dorsalis pedis artery is thus essential to understand avascular necrosis and triple arthrodesis of talus [10].

Dorsalis pedis flap is a hybrid axial/ arterial / myo -cutaneous flap available for treatment of defects in the distal part of the lower leg and heel region. It is thin and supple. Non palpable dorsalis pedis pulse with impaired blood flow as visualized by arteriography is a contraindication for the flap procedure [11]. Preoperative lower extremity imaging is advocated before fibular free flap transfer to identify atherosclerosis or absence of anterior tibial artery or its continuation, dorsalis pedis artery. The viability of the flap is thus entirely 
dependent on adequate vascularity of foot [12]. When peroneal artery reconstitutes the distal aspect of the hypoplastic anterior tibial artery, radial artery free flap is used instead of fibular flap because of risk of pedal ischemia [13].

First dorsal metatarsal artery, the branch of dorsalis pedis artery is dissected as a reverse - FDMA(First dorsal metatarsal artery) based skin flap for coverage of the toe defects. Lateral tarsal artery supplying extensor digitorum brevis serves as a pedicle for distal elevation of extensor digitorum brevis [14]. Lateral tarsal artery flap is an artery flap based on septocutaneous or musculocutaneous perforators supplied by the lateral tarsal artery. The external diameter of lateral tarsal artery is $1.42 \pm 0.34 \mathrm{~mm}$, length is $6.2 \pm 1.1$. $\mathrm{cm}$. This suitable vessel diameter, similar color and texture to hand, helps in its utility as a flap in repair of web contracture, a complication of hand injury [15].

The dorsalis pedis artery flap was first described by Mc Graw and Furlow in 1975 as a local transposition flap. It is utilized as a pedicled flap,island flap, free flap or distally based pedicled flap to cover defects of the distal foot after amputation of toes. However, the outcome of lower extremity reconstruction depends on variant anatomy and atherosclerotic disease [16].

A large pharyngocutaneous fistula occurring after cancer resection is closed with free dorsalis pedis flap after ablation of oesophageal cancer [16]. The dorsalis pedis flap is thus extensively used to reconstruct defects in the head and neck, upper and lower extremity. The features of flap include its thinness, vascularity, long vascular pedicle, inclusion of vascularized tendons such as extensor carpi brevis. The flap is also used for reconstruction of eye socket, palm and oral cavity [17].

$70 \%$ of diabetic patients present with a spectrum of peripheral vascular disease such as critical limb ischemia manifesting with intermittent claudication and rest pain .Signs of advanced ischemia include loss of hair growth over dorsum of toes and foot, thickening of toe nails, atrophy of skin and its appendages, delayed return of capillary blush, Buerger's sign(cadaveric pallor) and end stage is ulceration and gangrene. Arterial variations make palpation of the pedal pulse difficult. Absence of Dorsalis pedis artery pulsations is indicative of peripheral vascular disease/ vasculitis, collagen vascular disease, thrombotic disorders, congenital hypoplasia of dorsalis pedis artery, popliteal entrapment, aorto-iliac disease, Buerger's disease, Pseudo - occlusion of dorsalis pedis artery, diabetic autonomic neuropathy etc [18]. In addition, factors determining the pulsations include systolic perfusion pressure, arterial size, and amount of subcutaneous fat, oedema, fingertip insensitivity and ligamentum laciniatum [19]. Presence of pedal pulses rules out vascular insufficiencies. Awareness of arterial variations is germane for vascular surgeons during arterial reconstructions as in femorodistal bypass graft procedures and embolectomy. The precise knowledge of vascular anatomy of ankle is indispensible for surgical techniques such as ankle arthroscopy, surgical release of club foot etc. arterial abnormalities also increase the risk of postsurgical trauma; graft viability may be compromised [20].

Measurement of lower limb blood pressure is by ankle - brachial index(systolic blood pressure in brachial artery divided by systolic blood pressure in dorsalis pedis/ posterior tibial tibial artery), helpful for diagnosing peripheral vascular disease thus prediction of cardiovascular mortality and morbidity. Normal ankle brachial index is 0.9 to 1.1. Ankle brachial index of $<0.4$ indicates ischemic changes and $>0.9$ suggests stenosis of leg arteries and a very high value is suggestive of false assessment due to atherosclerotic stiffening [21].

Aneurysm of dorsalis pedis artery though rare $(0.5 \%)$ can lead to complications such as restricted ankle dorsiflexion due to involvement of deep peroneal nerve, localized distal ischemia of toes, contracture and gangrene.[22].Aneurysms of dorsalis pedis artery is clinically palpable as a pulsatile mass on dorsum of foot. It can cause compression of nerve with paraesthesia and pain [23].

\section{Conclusion}

Vascular anatomy of dorsalis pedis artery is essential for flap transfers as in bone and soft tissue repairs, reconstructions of foot as in setting of tumour resection, infection, trauma, to treat footischemiain diabetic patients. The anatomical basis for absence of its pulse is change in the arterial pattern and its small caliber. It is also an important landmark for micro vascular surgeries such as reimplantations and repairin the past few years, microvascular reconstruction techniques have become a routine to repair large softtissue defects related to trauma, non-healing wounds, chronic infection, sites of cancer, resection improving potential of limb salvage.

\section{Acknowledgements}

We extend our sincere thanks to our non - teaching staff, who helped us and supported during the writing of this manuscript. We also express our thanks to our institution, Dr. B.R. Ambedkar medical college and all the faculty members for helping in presentation of this manuscript. I "had full access to all the data in the study and I take responsibility for the integrity of the data and the accuracy of the data analysis." 


\section{References}

[1] Vijayalakshmi S, Gunapriya Raghunath and Varsha Shenoy, Anatomical study of Dorsalis pedis artery and its clinical correlations, Journal of clinical and diagnostic research, 5 (2), 2011, 287- 290.

[2] Kennedy Legel, Maxime Savard, Christopher J Blanco and Chaminda Jayanetti, Dorsalis pedis aneurysm: A case report and review of the literature, The foot and ankle Journal, 1 (11), 2008, 1.

[3] Rudolph S Reich,The pulses of the foot; Their value in the diagnosis of peripheral circulatory disease,Annals of surgery, 99(4), 1934,613-622.

[4] Maria Atanasova, Georgi P Georgiev and Lazar Jelev, Intriguing variations of the tibial arteries and their clinical implications, International journal of Anatomical variations, 4,2011, 45 - 47.

[5] Peter L.Williams and Roger Warwick, Gray's Anatomy(New york, Churchill Livingston, 36th edn 1980), $732,733,734$.

[6] Cronenwett, Gloviczki, Johnston, Krupski and Orriel et al, Rutherford Vascular surgery,1(Phladelphia,Elsevier Saunders,2005), 6th edn, 8,9,208,210t, 212,1258, 1259, 1260, 1187.

[7] Demetrios Chavatzas, Revision of the incidence of congenital absence of dorsalis pedis artery by an ultrasonic technique, Anatomical record, 178 (2), 2005, 289- 290.

[8] Stefen F Lange, Hans - Joachi Tramp.David Pittrow, Harald Darius and Mathias Malan, Profound influence of different methods for determination of the ankle brachial index on the prevalence estimate of peripheral arterial disease, BMC public health, 7,2007,147.

[9] Ertugrul Mavili, Halil Donmez, Guven Kahriman, Aysel Ozaslamaci, Nevzat Ozcan and Kutay Tasdemir, Popliteal artery branching patterns detected by digital subtraction angiography, Diagonostic Intervention Radiology, 2010, 3141-09.1

[10] G.L Mulfinger, Pasadena and J. Trueta, The blood supply of the talus, The Journal of bone and Joint surgery, 52 B (1), 1970, 160167.

[11] Christen Krag and Per Riegels- Nielsen, The dorsalis pedis flap for lower leg reconstruction , Acta Orthop Scand, 53 , 1982, 487493.

[12] Derek G Lohan, Anderanis. Tomasian, Mayil Krishnan, Praveen Jonnala and Keith.E Blackwell ,MR Angiography of Lower Extremities at 3 T: Presurgical Planning of Fibular Free FlapTransfer for Facial Reconstruction, American Journal of Roentgen Ray society, , 190, 2008, 770-776.

[13] Lawrence C Chow, Alessandro Napoli, Mathew B Klein, James Chang, Geoffrey and Rubin, Vascular mapping of the leg with multidetector Row CT Angiography prior to free flap transplantation, Radiology, 237, 2005, 353-360.

[14] C Balakrishnan, Y-J Chang, A Balakrishnan and D Careaga, Reversed dorsal metatarsal artery flap for reconstruction of a soft tissue defect of the big toe, Canadian Journal of Plastic Surgery, 17(3), 2009, 11-12.

[15] H Iang Dong, Wang Hong - gang, Zhao Cheng - yi and W Wei- zhi, An alternative approach in the treatment of thumb web contracture skin defects : lateral tarsal artery flap, Chinese Medical Journal, 122 (18), 2009,2133- 2137.

[16] Onder Tan, Bekir Atik and Duygu Ergen, Repair of Large Pharyngocutaneous fistula with the free dorsalis pedis flap, European Journal of General Medicine, 4 (1), 39-43 .

[17] R. Chitra, The relationship between the deep fibular nerve and the dorsalis pedis artery and its surgical importance, Indian Journal of plastic surgery, 42 (1), 2009, $18-21$.

[18] A. G. Unnikrishnan, Everyday Practice: Diabetes MellitusApproach to a patient with a diabetic foot, The National medical Journal of India, 21(3), 2008,134-137.

[19] A Mowlavi, J White man, B.J Welhalm, M.W Neumeister and R MC Lafferty, Dorsalis pedis artery palpation using a bony landmark, Postgraduate Medical Journal ,78, 2002,746-747.

[20] Ashok Raj Koul, Rahul K Patil and Vinoth Kumar Philip, Coverage of defects over toes with distally based local flaps: A report of four cases, Indian Journal of Plastic Surgery, 41 (1), 2008,62-66

[21] Noritaka Isogai, William Landis, Tae ho kim, laus C Gerstenfeld and Joseph Uptor et al, Formation of Phalanges and small joints by Tissue engineering, Journal of Bone \& Joint Surgery, 81,1999, 306-16.

[22] Ashok L Ramavath, Julie A Cornish, Muthu Ganapathi and Dean T Williams, Missed diagnosis of ankle pseudo aneurysm following ankle arthroscopy: a case report, Cases Journal,2,2009, 162.

[23] Robert L Ruberg and David J Smith, Plastic surgery, A Core Curriculum,(New York, Mosby Pub 1994), 52, 53, 77, 594, 613. 